\title{
Synthesis and Physicochemical Behaviour of Polyurethane-Multiwalled Carbon Nanotubes Nanocomposites Based on Renewable Castor Oil Polyols
}

\author{
Alaa Ali, ${ }^{1,2}$ Kamal Yusoh, ${ }^{1}$ and S. F. Hasany ${ }^{1}$ \\ ${ }^{1}$ Faculty of Chemical \& Natural Resources Engineering, Universiti Malaysia Pahang, 26300 Kuantan, Malaysia \\ ${ }^{2}$ Department of Chemical Engineering, University of Technology, Baghdad, Iraq \\ Correspondence should be addressed to Alaa Ali; allavip63@yahoo.com
}

Received 7 May 2014; Accepted 28 August 2014; Published 13 October 2014

Academic Editor: Shafiul Chowdhury

Copyright (C) 2014 Alaa Ali et al. This is an open access article distributed under the Creative Commons Attribution License, which permits unrestricted use, distribution, and reproduction in any medium, provided the original work is properly cited.

Polyurethanes (PUs) are high performance materials, with vast industrial and engineering applications. In this research, effects of Multiwalled Carbon Nanotubes (MWCNTs) on physicochemical properties of Castor Oil based Polyurethanes (COPUs) were studied. MWCNTs were added in different weight percentages ( $0 \%$ to $1 \% \mathrm{wt}$ ) in a castor oil based polyurethane (COPUs-MWCNTs) nanocomposites. The composition, structure, and morphology of polyurethanes were characterized by Fourier transform infrared spectroscopy (FTIR), X-ray diffraction (XRD), scanning electron microscopy (SEM), field emission scanning electron microscopy (FESEM), and element detection by energy dispersive spectroscopy (EDX) analysis, respectively. Thermal stability was studied by thermogravimetric analysis (TGA). Barrier properties and surface area studies were investigated by nitrogen permeability machine and BET technique. Mechanical properties were calculated by tensile universal testing machine. Results showed well dispersed MWCNTs in polyurethane matrix at different weight percentages. The best results were obtained with $0.3 \mathrm{wt} \%$ of MWCNTs in the composite. Surface area studies revealed presence of very few pores which is in a good agreement with barrier permeability, reduced up to $\sim 68 \%$ in $1 \mathrm{wt} \%$ and $~ 70 \%$ in $0.5 \mathrm{wt} \%$ of MWCNTs in polymer matrix, with respect to pure COPUs samples.

\section{Introduction}

Polyurethanes are versatile polymeric materials with extensive demand due to exceptional physical properties (e.g., high flexibility, high tensile strength, tear and abrasion resistance, solvent resistance, etc.) and high versatility in chemical structures (discussed by Lu and Macosko [1]; reported by Cao et al. [2]; studied by Oprea [3]; discussed by Akintayo et al. [4]). Footwear, machine industry, coatings and paints, rigid insulations, thermoplastic, foams, and medical devices (discussed by Yusoh et al. [5]; reported by Krushna and Nayak [6]; and discussed by Bhuva Bharat [7]) are some important industrial applications. Polyurethanes are generally synthesized by the polyols derived from the petrochemical industry (reported by Kong et al. [8]; discussed by Lligadas et al. [9]; and studied by [10]). The synthesis involves a poly addition polymerization reaction between organic isocyanate and polyol.

Due to large consumption of PUs, a huge demand of utilization of renewable resources is always a critical job for researchers, to overcome environmental and price controlling issues related with a petroleum industry (discussed by Fan et al. [11]; reported by Spontón et al. [12]). Chang and $\mathrm{Lu}$ [13] depicted novel polyols made from modified castor oil (MCO) that was synthesized using a transesterification process with the glycerol competing with the petroleum polyols to overcome the environmental concerns and rising prices of the mineral oil industry. The commercial utilization of biodegradable polymers has become an active research area during past decades due to potential advantages compared with synthetic petroleum polymers owing to their biodegradable properties (discussed by Xu et al. [14]; studied by Baruah [15]). Yuan [16], in his thesis, presented polyol derived from soybean oil; however, this has some major disadvantages that limited its applications. Polyurethane based on polyols derived from different vegetable oils, like castor (discussed by Yeganeh and Hojati-Talemi [17]; studied by Corcuera et al. [18]), soybean (reported by Ismail et al. [19]; discussed by 
Yang et al. [20]; studied by Bakhshi et al. [21]), sunflower (discussed by Das et al. [22]), and rapeseed oils (studied by Rojek and Prociak et al. [23]; discussed by Fridrihsone et al. [24]; reported by More et al. [25]) with or without modifications, were being used these days due to their excellent properties derived from the hydrophobic nature of triglycerides.

Castor oil possesses a reactive hydroxyl $(-\mathrm{OH})$ functional group contained in its structure that can be used as a polyol and so it is widely used in many chemical industries, especially in the production of polyurethanes by reacting with different diisocyanates, with large availability, nonedibility, renewability, high purity, biodegradability, and low cost (reported by Spontón [12]; discussed by Carme Coll Ferrer et al. [26]). Ivan et al. [27] investigated the properties of environmentally friendly castor oil, based on polyurethane (COPU) hybrid materials with titanium (IV) oxide nanoparticles, as filler, and different types of diisocyanate (toluene diisocyanate and isophorone diisocyanate). Dos Santos et al. [28] presented the preparation and diagnosis of diisocyanate groups from castor oil. Their results exhibited that increasing polyol amount leads to an increase in the strength at rupture of the obtained polymers and a decrease in the polymers elongation, which results into increased modulus. Yeganeh and Hojati-Talemi [17] studied the synthesis of polyurethane networks based on castor oil as a renewable resource of polyol and poly(ethylene glycol) (PEG) through the reaction of epoxy-terminated polyurethane prepolymer (EPUs) with 1,6hexamethylene diamine, for biomedical majoring. The results found that mechanical properties of the composites were improved by increasing EPUs content, as a result of which surface and bulk properties increase which also increased the hydrolytic degradation rate consequently.

Carbon nanotubes (CNTs) represent the ideal, most perfect, and ordered carbon fiber, the structure of which is entirely known at the atomic level and distinctly different from the structure of traditional carbon fibers (discussed by Endo et al. [29]) resulting in the unique properties such as superconductivity, light weight, high stiffness, and axial strength (reported by Kurahatti et al. [30]; discussed by Luo et al. [31]). CNTs, because of their high mechanical strength, are being considered as nanoscale fibers to enhance the performance of polymer nanocomposite materials (studied by Yadav and Cho [32]). Madkour et al. [33] studied the synthesis of thermoplastic polyurethane (TPU), reinforced with multiwalled carbon nanotubes with better thermal stability and improved mechanical performance of thermoplastic polyurethane (TPU). Yadav and Cho [32] illustrated mechanically robust antimicrobial nanocomposites of multiwalled carbon nanotubes nanofillers and hyperbranched polyurethane (HBPU). Yun et al. [34] investigated PU grafted multiwalled carbon nanotubes (PU-g-MWCNTs) to fabricate electroconducting nanocomposites. All of these studies were based on petroleum originated polyols; no study has been reported so far on green and renewable sources of polyols.

In this study, the COPUs-MWCNTs polymer nanocomposite has been designed based on green and renewable castor oil as a polyol source, reinforced with purified MWCNTs to investigate the physicochemical behaviour of the synthesized polymer composites. In situ polymerization technique assisted by ultrasonication was adopted with different dispersion times of MWCNTs in the composite matrix to accomplish enhanced dispersion in the reacting mixture.

\section{Experimental}

2.1. Materials. MWCNTs used in this study have the following specifications: purity $>90 \%$; outer diameter, $30-50 \mathrm{~nm}$; inner diameter $5-10 \mathrm{~nm}$; and length $10-20 \mu \mathrm{m}$. They were obtained from Chengdu organic chemicals, China. Castor oil was of commercial grade and was purchased from the local market. It was dehydrated at $80^{\circ} \mathrm{C}$ in a vacuum oven and characterized for hydroxyl value (148), acid value (2), and moisture content $(0.379 \%)$. Polypropylene glycol (PPG) $(\mathrm{Mn}=$ 4000) was supplied by SIGMA-Aldrich Company. Chain extender, 1,4-butanediol, was procured from Himedia, India. The toluene diisocyanate (TDI) and dimethylformamide (DMF) which were used as received were supplied from SIGMA-Aldrich Company.

2.2. Purification of $M W C N T$ s. Initially, as provided, MWCNTs were preheated at $\sim 450^{\circ} \mathrm{C}$ for 30 minutes to remove amorphous carbon (discussed by Manivannan et al. [35]). The MWCNTs were purified via stirring using an acid treatment ( $50 \mathrm{~mL}$ of $95 \% \mathrm{H}_{2} \mathrm{SO}_{4}$ and $20 \mathrm{~mL}$ of $65 \% \mathrm{HNO}_{3}$ ), with a ratio of $(3: 1)$, at $80^{\circ} \mathrm{C}$ for 5 hours, and this was followed by filtration (studied by Yun et al. [34]; discussed by Jana et al. [36]; reported by Yudianti et al. [37]). The collected solid was washed out with distilled water till $\mathrm{pH}$ neutralized $(\sim 7 \mathrm{pH})$. The filtered solid was then washed with $25 \mathrm{~mL}$ of acetone to remove most of the water from the sample and dried at $80^{\circ} \mathrm{C}$ for 20 hours. The purity of the MWCNTs was confirmed by FTIR spectroscopy.

\subsection{Synthesis of MWCNTs-Polyurethane (COPUs-MWCNTs)} Nanocomposites. Purified MWCNTs reinforced polyurethane nanocomposites were fabricated via in situ polymerization. The synthesis process involved two stages; the initial dispersion is followed by mixing of raw materials as follows. Firstly, different weight percent of the functionalized MWCNTs $(0.1,0.2,0.3,0.4,0.5$, and $1 \mathrm{wt} \%)$ was dispersed by blending the MWCNTs in a mixture of polypropylene glycol polyol and dehydrated castor oil $(15 \%)$ at $80^{\circ} \mathrm{C}$, followed by ultrasonic bath for up to three hours at $50^{\circ} \mathrm{C}$.

In the second stage, the mixture was dissolved with $6.5 \mathrm{gm}$ of toluene diisocyanate (TDI) drop-by-drop and vacuum degassed for 3-5 minutes at $85^{\circ} \mathrm{C}$ to achieve homogenous reaction and to prevent the bubbles formation. Prepolymer obtained was lastly reacted with $0.9 \mathrm{gm}$ of 1,4 -butanediol in the presence of $0.25 \mathrm{gm}$ catalyst (DABCO) at $85^{\circ} \mathrm{C}$ for two minutes; then $50 \mathrm{~mL}$ of dimethylformamide (DMF) was added gradually and the temperature was reduced to $70^{\circ} \mathrm{C}$. The mixture was continuously stirred until solution reduced up to $40 \%$ and became viscous; it was then poured into a mould to obtain a thin film and then cured in an oven at $65^{\circ} \mathrm{C}$ under vacuum for 24 hours. Obtained film was later postcured for a week at $80^{\circ} \mathrm{C}$. 


\section{Characterization}

The crystal structure of the COPUs-MWCNTs nanocomposites was analysed by X-ray diffraction (XRD), using an Xray diffractometer (Rigaku Miniflex II, Japan) employing a graphite monochromator and $\mathrm{CuK} \alpha$ radiation $(\mathrm{lnm})$. Fourier transform infrared spectroscopy (FTIR) analysis of COPUsMWCNTs nanocomposites was done using a FTIR spectrometer (Nicolet 5DX FT-IR, USA). The morphology of the hybrids was examined by field emission scanning electron microscopy (FE-SEM; JEOL EVO-50, Japan) and scanning electron microscopy (SEM; JEOL 6300F, Japan). Element detection EDX was performed with the attached equipment in FESEM spectroscopy. Thermal stability of polyurethane nanocomposites was determined using a thermogravimetric analyzer (TGA) type Universal V4.5A, TA instruments were under nitrogen atmosphere. Tensile testing was carried out on an Instron model 4505 universal testing machine at $25^{\circ} \mathrm{C}$, with a load cell of $5 \mathrm{KN}$ and following ASTM D 638. The crosshead speed was set to $2 \mathrm{~mm} / \mathrm{min}$. Samples were cut in a dumbbell shape with an ASTM D 638 (type V). Surface area properties were studied by BET Micromeritics surface area analyzer and gas permeability test was done by nitrogen permeable machine setup.

\section{Results and Discussion}

4.1. Structure and Morphology of PU Nanocomposites. FTIR spectrum indicates a peak at ranges from 1715 to $1725 \mathrm{~cm}^{-1}$, which refers to carbonyl hydrogen bonding present in nanocomposites, with different intensities (discussed by Jana et al. [36]); the COPUs peak is much sharper and intense while in the case of both of the COPU nanocomposites the peak became broad with lower intensity, predicting a reduction in carbonyl hydrogen bonding due to purified MWCNTs intercalation in COPU matrix. From Figure 1, one characteristic peak of MWCNTs and two characteristic peaks of COPUs were observed at near $3500 \mathrm{~cm}^{-1}$ (reported by Yudianti et al. [37]), $3340 \mathrm{~cm}^{-1}$ (discussed by Yadav et al. [38]), and $1718 \mathrm{~cm}^{-1}$ (reported by Gu et al. [39]) due to the stretching vibration of $-\mathrm{OH},-\mathrm{NH}$, and $-\mathrm{C}=\mathrm{O}$ groups in purified MWCNTs and COPUs, respectively. It was clearly seen that the $\mathrm{OH}$ peak intensity at $3500 \mathrm{~cm}^{-1}$, which shows the strongest peak in the MWCNTs, vanishes with the addition of MWCNTs in polymer; Sahoo et al. [40] signified that MWCNTs were successfully covalently encapsulated by two layers of polymer. In this study, the MWCNTs used in the composite preparation were an acid-treated carbon nanotube which contains carboxylic acid groups. The carboxylic acid groups may have reacted with $\mathrm{OH}$ groups of the polymer to form ester groups (discussed by Wang et al. [41]).

Figure 2 depicts the diffraction peaks of the pure COPUs and the COPUs-MWCNTs nanocomposites at $2 \theta=20.2^{\circ}$, $20.1^{\circ}$, and $20^{\circ}$ of the reflection plane with interchain $(d)$ spacing of $4.445,4.451$, and $4.501 \mathrm{~A}^{\circ}$, respectively, which is related to the existence of short range regular ordered structure of both the hard and the soft domains along with disordered structure of amorphous phase of the polyurethane

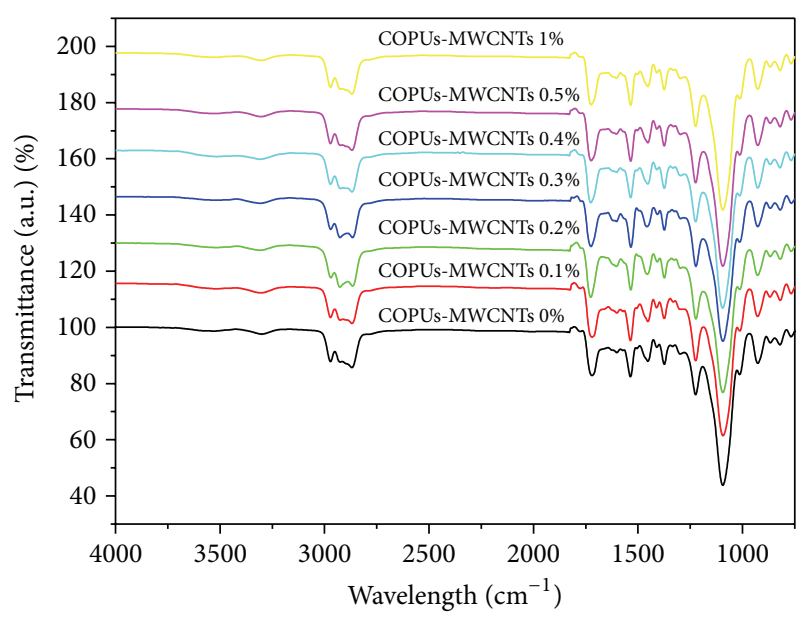

FIGURE 1: FT-IR spectra of pure COPUs and COPUs-MWCNTs nanocomposites, with virgin $0.1,0.2,0.3,0.4,0.5$, and $1 \mathrm{wt} \%$ MWCNTs loadings.

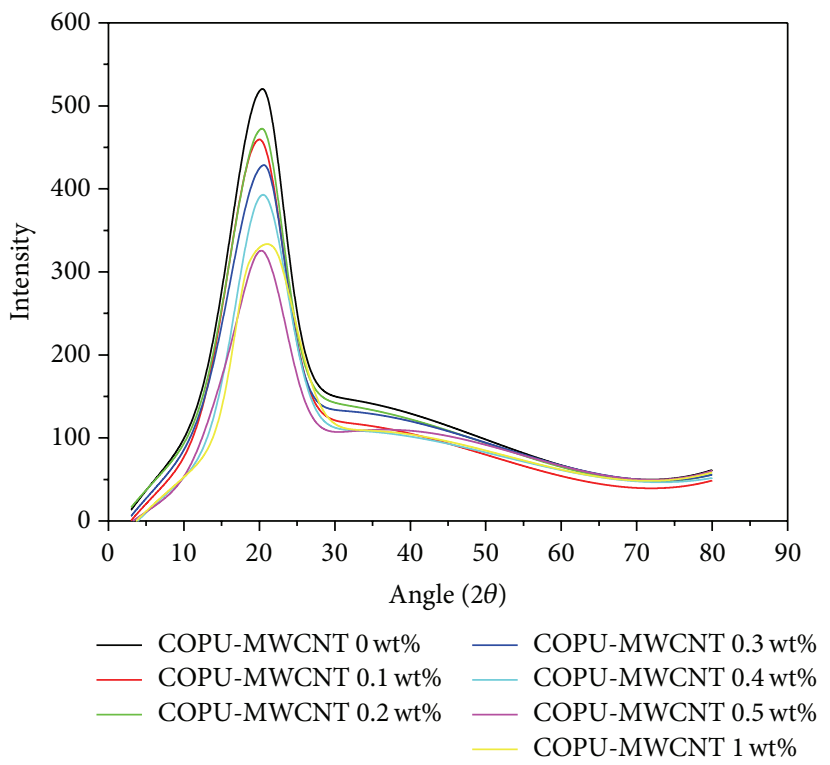

FIgURE 2: Powder X-ray diffraction patterns of pure COPUs and COPUs-MWCNTs nanocomposites, with virgin $0.1,0.2,0.3,0.4,0.5$, and $1 \mathrm{wt} \%$ MWCNTs loadings.

matrix. Peaks become broadened with reduced intensity, with the addition of MWCNTs, which inferred that the MWCNTs considerably affect the well short-range microstructural phases of both soft and hard segments of the COPUs matrix (reported by Aruna and Deba [42]; discussed by Kuan et al. [43]). This may be due to the presence of strong interfacial interactions between MWCNTs and COPUs matrix. In addition, the individual or bundled nanotubes also influence the resulting steric hindrance effect of the soft and the hard phases of COPUs matrix (discussed by Jana et al. [36]). Peak assigned to the (100) plane of the MWCNTs was found at $2 \theta$ value $\sim 40^{\circ}$ (100) Bragg reflection planes. The peaks are attributed to the ordered regular arrangement of 


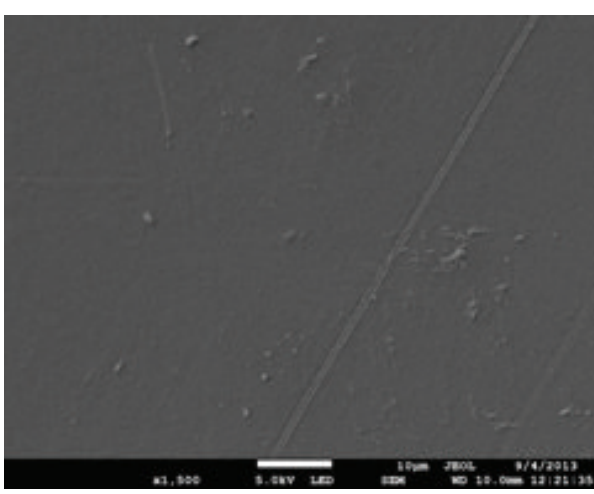

(a)

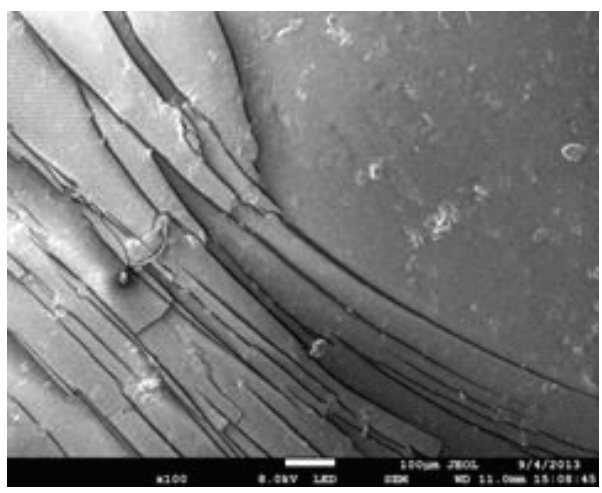

(c)

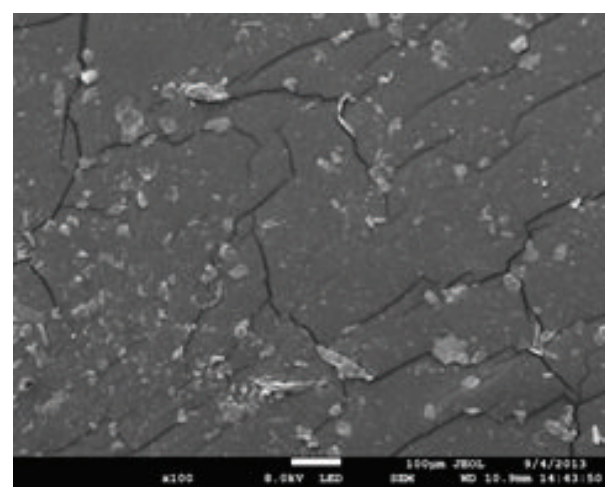

(b)

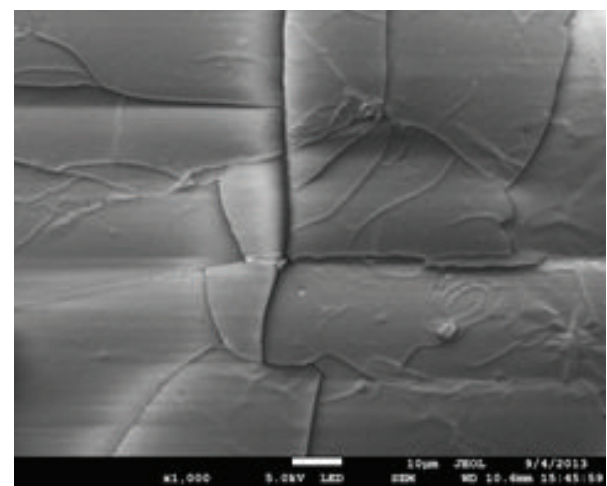

(d)

FIGURE 3: FESEM images of fractured surface of (a) $0 \%$ MWCNTs, (b) $0.3 \%$ MWCNTs, (c) $0.5 \%$ MWCNTs, and (d) $1 \%$ MWCNTs in COPUs matrix.

the concentric cylinders of the graphitic carbon atoms in nanotubes (studied by Zhou et al. [44]).

Surface morphology of the pure COPUs and MWCNTs reinforced COPUs nanocomposites at $\times 50,000$ and $\times 200,000$ magnifications is shown in Figure 3 and is studied by FESEM microscopy. The bright dots and tread-like structures in the images are attributed to the MWCNTs. The dispersion of MWCNTs in the COPUs matrix at lower loading $(0.3 \mathrm{wt} \%)$ as shown in Figure 3(b), is found to be homogeneously dispersed throughout the COPUs matrix without any agglomeration. But agglomeration is seen at higher (0.5 and1 wt\%) loadings depicted in Figures 3(c) and 3(d), respectively. In $0.3 \mathrm{wt} \%$ MWCNTs loading micrographs, well-dispersed nanotubes throughout the entire surface could be attributed to the induced Van der Waals force between polymer and MWCNTs, which exceeds the existing intertube forces during sonication (discussed by Ma et al. [45]).

The result is in good agreement with the thermogravimetric (TGA) results, where $0.3 \mathrm{wt} \%$ MWCNTs showed higher stability than other percentages. In micrographs (0.5 and $1 \mathrm{wt} \%)$, high agglomeration of MWCNTs is seen, which may be due to self-aggregation of MWCNTs by Van der Waals bonding and also due to lack of interfacial adhesion between MWCNTs and COPUs matrix (reported by Aruna and Deba [42]; investigated by Nayak et al. [46]).

Figure 4 shows SEM images of the fractured surfaces of pure and COPUs-MWCNTs nanocomposites in different weight percentages. The homogeneous dispersion of MWCNTs in the polymer matrix is one of the conditions needed for a composite to display good mechanical strength reinforcement because inhomogeneities can lead to structural defects in the composite material (investigated by Yoo et al. [47]). The bright dots and thread-like structures in the images are attributed to the MWCNTs in composite Figures 4(a), 4(b), 4(c), 4(d), 4(e), and 4(f), where some microaggregates inside the film are present which might be the result of DMF evaporation which decreases the total solubility of solution and increases the difference of the solubility of the solution and the nanotubes (discussed by Wongtimnoi et al. [48]).

The dispersion of MWCNTs in the COPUs matrix up to $0.3 \mathrm{wt} \%$ MWCNTs, as shown in Figure $4(\mathrm{~d})$, is found to be homogeneously dispersed throughout the COPUs matrix with no or very little agglomeration, which is quiet prominent at higher $(0.4$, and $0.5 \mathrm{wt} \%)$ loadings as depicted in Figures 4(e) and 4(f), respectively. In $0.3 \mathrm{wt} \%$ MWCNTs loading micrographs, well-dispersed nanotubes throughout the entire surface could be attributed to the induced Van der Waals force between polymer matrix and MWCNTs, which surpasses the standing intertube forces during sonication (discussed by Ma et al. [45]). The better dispersion of the MWCNTs results in more effective transfer of load imposed on the COPUs-MWCNTs nanocomposite and higher effective filling volume, which can be contributed more likely to 


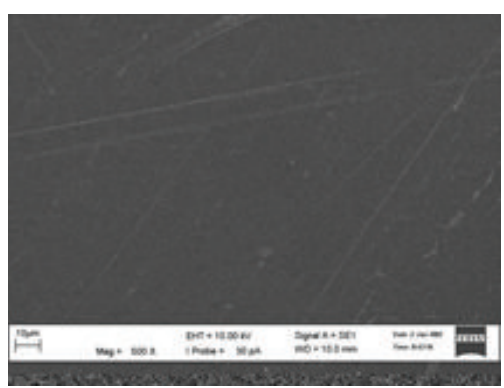

(a)

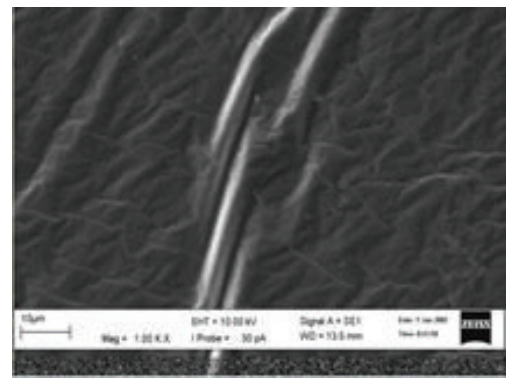

(d)

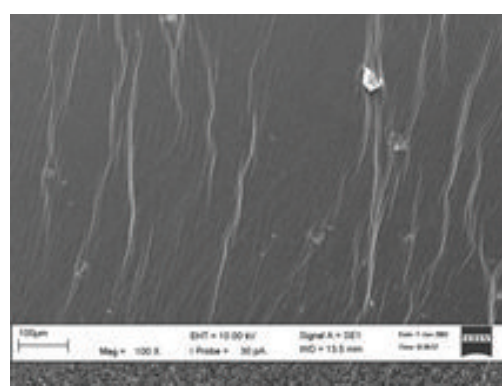

(b)

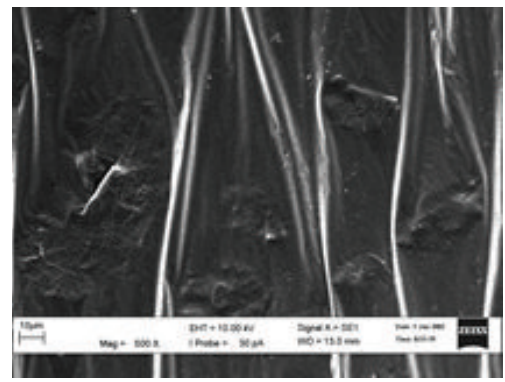

(e)

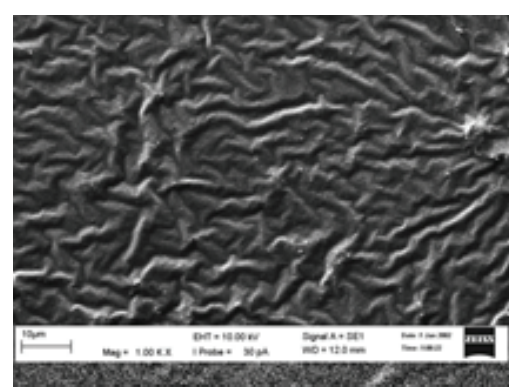

(c)

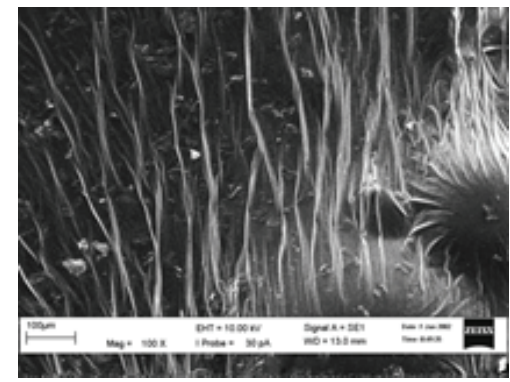

(f)

FIgURE 4: SEM images of pure COPUs and COPUs-MWNTs composites: (a) COPUs, (b) COPUs-MWNTs 0.1\%, (c) COPUs-MWNTs 0.2\%, (d) COPUs-MWNTs $0.3 \%$, (e) COPUs-MWNTs $0.4 \%$, and (f) COPUs-MWNTs $0.5 \%$.

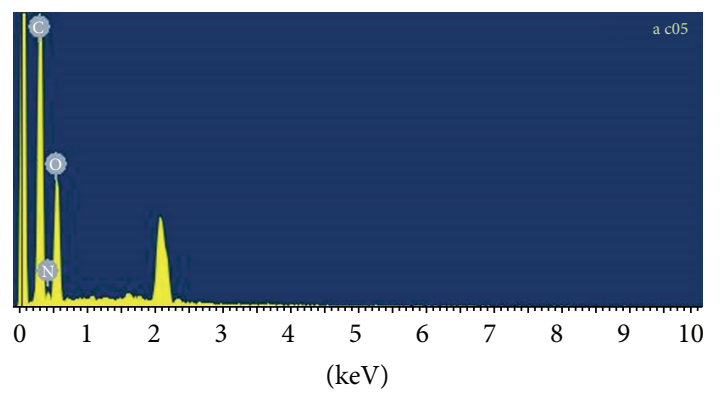

Full scale 1434 cts crusor: 0.000

FIGURE 5: EDX analysis of COPUs-MWCNTs nanocomposites.

the improvement of mechanical properties (reported by $\mathrm{Gu}$ et al. [39]).

4.2. Element Detection Analysis. The purity and elemental composition of the synthesized polyurethane-MWCNTs nanocomposites were confirmed by the EDX measurement as shown in Figure 5. EDX analysis confirms that only Oxygen $(\mathrm{O})$, Carbon $(\mathrm{C})$, and Nitrogen $(\mathrm{N})$ elements are present in the nanocomposites, which is in good agreement with the stoichiometric composition of polyurethane-MWCNTs nanocomposites. $\mathrm{O}, \mathrm{C}$, and $\mathrm{N}$ are the main elements of polyurethane-MWCNTs nanocomposites and carbon peak belongs to both of COPUs and MWCNT. The EDX elemental microanalysis (mass percentage) of nanohybrid is listed in Table 1.
TABLE 1: EDX studies of different weight\% of MWCNTs in COPU matrix with elemental weight and atomic percentages.

\begin{tabular}{lccc}
\hline $\begin{array}{l}\text { MWCNTs } \\
\text { wt\% }\end{array}$ & Element & $\begin{array}{c}\text { Weight } \\
\text { percentage }\end{array}$ & $\begin{array}{c}\text { Atomic } \\
\text { percentage }\end{array}$ \\
\hline \multirow{3}{*}{0.5} & $\mathrm{C}$ & 59.85 & 65.73 \\
& $\mathrm{~N}$ & 9.85 & 9.28 \\
& $\mathrm{O}$ & 30.3 & 24.99 \\
\hline \multirow{3}{*}{0.3} & $\mathrm{C}$ & 59.80 & 65.25 \\
& $\mathrm{~N}$ & 9.84 & 9.3 \\
\hline \multirow{2}{*}{0.1} & $\mathrm{O}$ & 30.9 & 24.45 \\
& $\mathrm{C}$ & 59.78 & 65.05 \\
& $\mathrm{~N}$ & 9.82 & 9.35 \\
& $\mathrm{O}$ & 30.4 & 25.6 \\
\hline
\end{tabular}

4.3. Thermogravimetric Analysis (TGA). Thermogravimetric behavior is a good evidence of the interactions within the COPUs-MWCNTs nanocomposites. In this study thermogravimetric stability was calculated on different percentages of MWCNTs $(0.1,0.2,0.3,0.4,0.5$, and $1 \mathrm{wt} \%)$ as shown in Figure 6 within the COPU matrix, which was previously compared with the neat polyurethane. The samples are heated from room temperature to $550^{\circ} \mathrm{C}$ at a rate of $10^{\circ} \mathrm{C} / \mathrm{min}$ under inert atmosphere. The temperature of pure polyurethane is $\sim 341.2^{\circ} \mathrm{C}$ (discussed by Kuan et al. [43]). The better result is obtained with $0.3 \mathrm{wt} \%$ of MWCNTs in the COPU matrix. In the COPUs-MWCNTs nanocomposites, decomposition temperature of polyurethane part is observed at $\sim 410^{\circ} \mathrm{C}$ with a weight loss of $\sim 89 \%$. The decomposition temperature is 


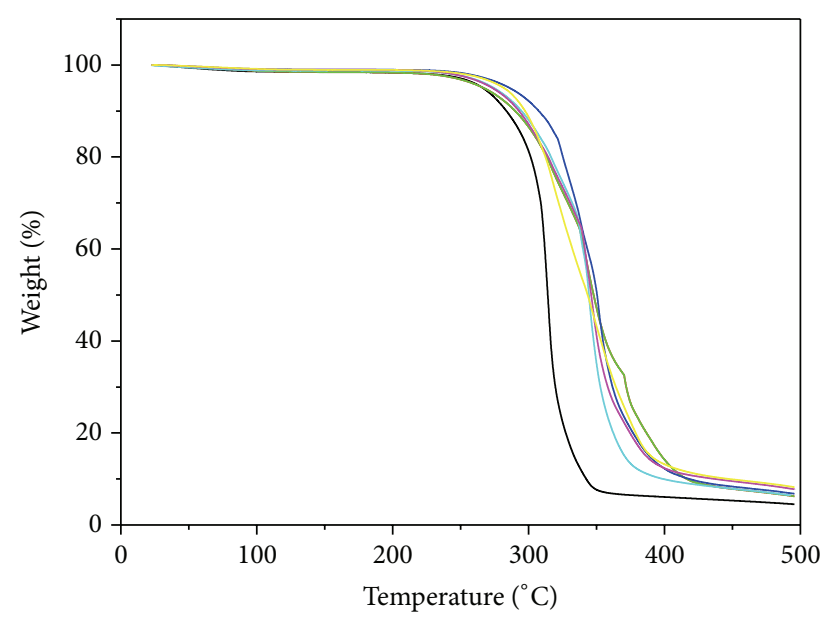

- COPUs-MWCNTs 0\% COPUs-MWCNTs $0.1 \%$ COPUs-MWCNTs $0.2 \%$

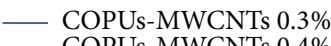

COPUs-MWCNTs $0.4 \%$ COPUs-MWCNTs $0.5 \%$ COPUs-MWCNTs $1 \%$

Figure 6: TGA spectrum of pure COPUs and COPUs-MWCNTs nanocomposite with varying MWCNTs wt $\%$.

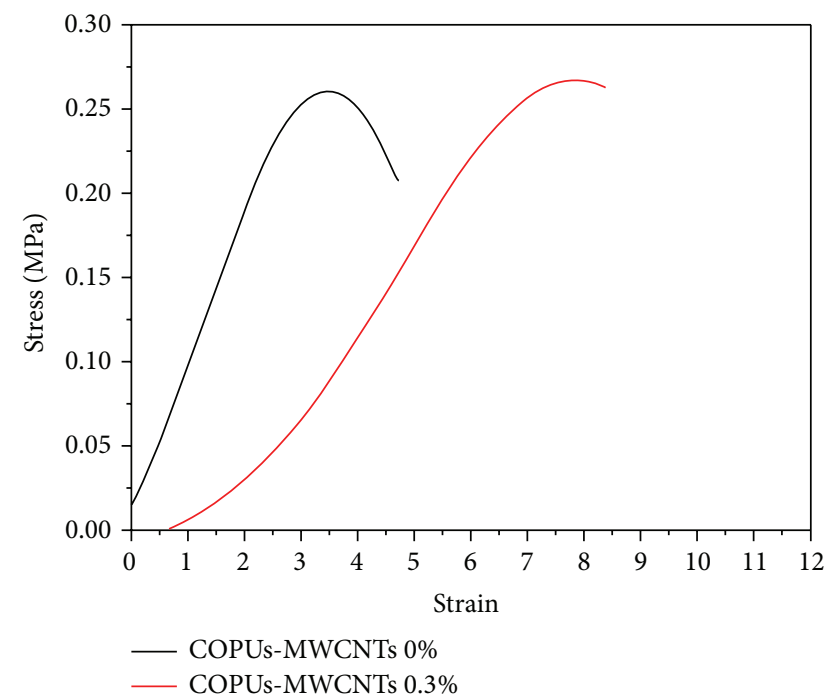

FIGURE 7: Stress-strain curves of pure COPUs and COPU-MWCNTs $0.3 \mathrm{wt} \%$ composites.

increased up to $\sim 50^{\circ} \mathrm{C}$ with the addition of MWCNTs, which may be ascribed to the combined effect of excellent thermal stability of MWCNTs and their strong interfacial interactions within the layers of polymer matrix (investigated by Guo et al. [49]). This also provides the quantitative evidence of $11 \%$ in weight of MWCNTs present in the sample of polymer matrix.

4.4. Mechanical Properties of PU Nanocomposites Film. A comparative stress-strain study of pure COPUs and COPUsMWCNTs nanocomposites with $0.3 \mathrm{wt} \%$ MWCNTs depicts an improvement of $\sim 5 \%$ in tensile strength and $\sim 128 \%$ in modulus in COPUs-MWCNTs nanocomposites as shown in Figures 7 and 8. The dispersion of MWCNTs into the COPUs

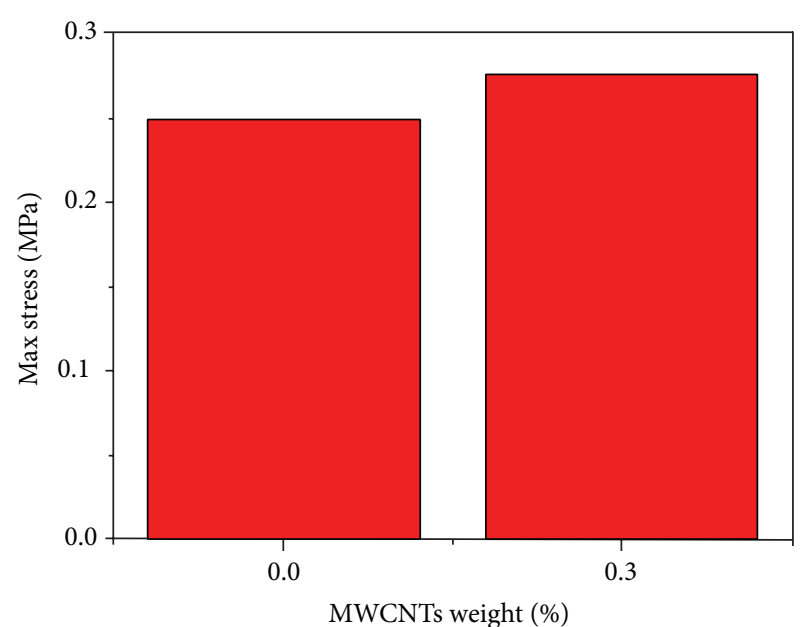

FIGURE 8: Tensile strength of COPUs and COPUs-MWCNTs nanocomposites with pure and $0.3 \mathrm{wt} \%$ MWCNTs.

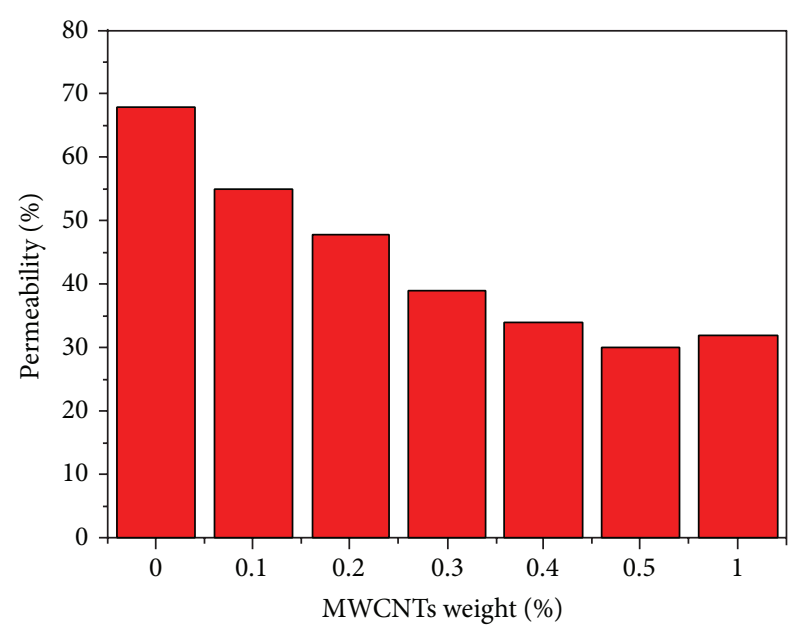

FIGURE 9: Nitrogen permeability rate in different MWCNTs weight percentages.

matrix increases the resistance of the polymer to deformation. This is due to well-dispersed and polymer-filler interaction in the nanocomposites (discussed by Jana et al. [36]). The main reason for this improvement can be ascribed to COPUs-MWCNTs hitching and hydrogen bonding between MWCNTs particles and the polymer (reported by Krushna and Nayak [6]). It may be also because of carboxylic groups located onto the MWCNTs surface which form covalent bonds with the COPUs resulting in significantly enhanced interfacial adhesion (investigated by Sahoo et al. [50]).

4.5. Gas-Barrier Properties. The inclusion of MWCNTs into the COPUs matrix has a huge impact on the gas diffusion mechanism due to different permeability properties of the matrix and the MWCNTs nanofiller (discussed by Maji et al. [51]). A reduction in permeability of nitrogen is observed with an increase in MWCNTs concentration (Figure 9). Samples up to $0.5 \mathrm{wt} \%$ of MWCNTs show permeability reduction, 


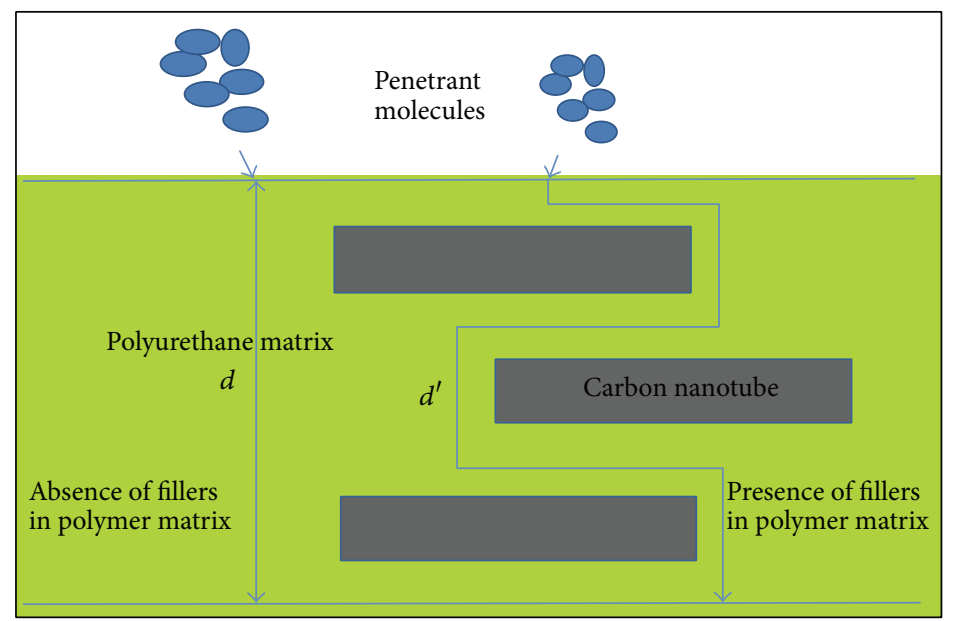

FIGURE 10: Schematic model describing the path of the diffusing $\mathrm{N}_{2}$ gas through the COPU-MWCNTs nanocomposite. (d) is the actual distance travelled in the absence of fillers and $\left(d^{\prime}\right)$ is tortuous path length in the presence of fillers.

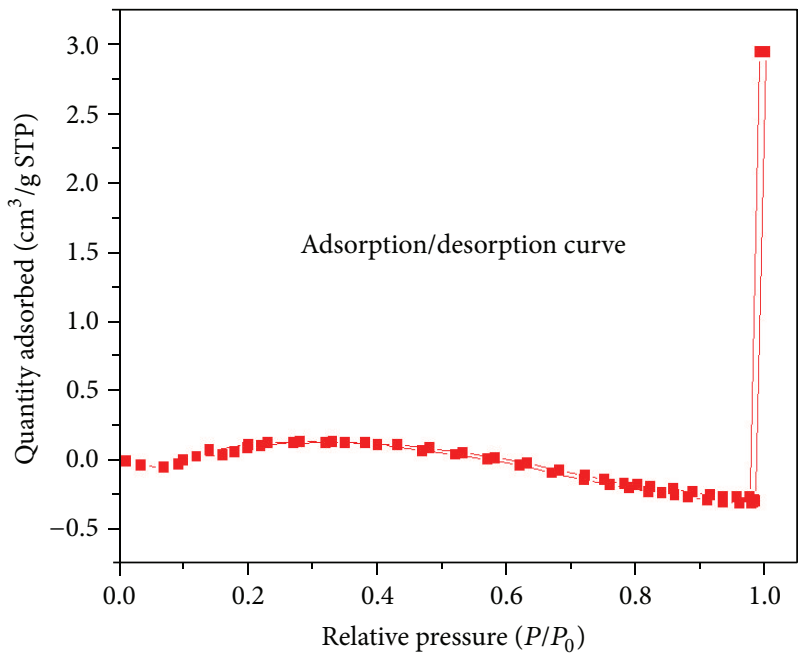

FIGURE 11: Nitrogen adsorption-desorption isotherms of $0.5 \mathrm{wt} \%$ COPU-MWCNTs.

which is $\sim 70 \%$ over the pure COPU, but beyond that, the decrease is insignificant (for $1 \mathrm{wt} \%$ loading, the reduction is $\sim 68 \%$ ). The increase in gas barrier property for polymerMWCNTs nanocomposite may be due to the exfoliation, compatibilization, orientation, and reaggregation of purified MWCNTs in to the polymer matrix, as shown in Figure 10 (studied by Joshi et al. [52]).

4.6. BET Surface Analysis. BET surface area analysis is investigated on $0.5 \mathrm{wt} \%$ COPUs-MWCNTs (Figure 11) nanocomposites by nitrogen adsorption/desorption isotherms measured at $77 \mathrm{~K}$ and data are treated according to the BrunauerEmmett-Teller (BET) theory investigated by Brunauer et al. [53]. The surface area is determined by a multipoint BET method using the adsorption data in the relative pressure $\left(P / P_{0}\right)$ range of $0.05-0.3$. The BET surface area, obtained for sample, is $0.3845 \mathrm{~m}^{2} \mathrm{~g}^{-1}$. This value is considerably low, which is in a good agreement with the barrier properties indicating presence of very low or no pores in the surface resulting in low permeability that may be due to the exfoliation, compatibilization, orientation, and reaggregation of purified MWCNTs in to the polymer matrix.

\section{Conclusions}

In the conclusion, castor oil based polyurethanes nanocomposites (COPUs-MWCNTs) are synthesized with different weight percentages of MWCNTs. The thermal and mechanical properties of the COPUs matrix are significantly enhanced by the incorporation of MWNTs. FTIR, of the nanocomposites, shows broad peaks with higher intensity, with the increase in MWCNTs weight percentage in the polymer. It indicates a good physical entanglement between the constituents. XRD results revealed broad peaks with lower intensity in relation with pure COPUs, which inferred that the MWCNTs considerably affect the well short-range microstructural phases of both the soft and the hard segments of the COPUs matrix, resulting in strong interfacial interactions between MWCNTs and COPUs matrix. FESEM showed well dispersed MWCNTs in the COPUs matrix up to $0.3 \mathrm{wt} \%$, but agglomeration is seen at higher (0.5 and $1 \mathrm{wt} \%)$ loadings. Thermogravimetric analysis depicts that the $0.3 \mathrm{wt} \%$ of MWCNTs in the polymer has best thermal stability in relation with other percentages and neat polyurethane, which is in total agreement with FESEM results. SEM monographs displayed good dispersion up to $0.3 \mathrm{wt} \%$ loaded MWCNTs in the polymer matrix, which is in good relation with FESEM and TGA results. EDX analysis showed presence of only carbon, nitrogen, and oxygen in different weight and atomic percentages. The barrier properties show improvement with increasing MWCNTs in the polymer matrix up to 5\%, which was found compatible with low surface area calculated by BET surface analysis. 


\section{Conflict of Interests}

The authors declare that there is no conflict of interests regarding the publication of this paper.

\section{Acknowledgment}

Authors are thankful to Universiti Malaysia Pahang for research Grant RDU 130329 and for the financial support.

\section{References}

[1] Q. W. Lu and C. W. Macosko, "Comparing the compatibility of various functionalized polypropylenes with thermoplastic polyurethane (TPU)," Polymer, vol. 45, no. 6, pp. 1981-1991, 2004.

[2] X. Cao, L. James Lee, T. Widya, and C. Macosko, "Polyurethane/ clay nanocomposites foams: processing, structure and properties," Polymer, vol. 46, no. 3, pp. 775-783, 2005.

[3] S. Oprea, "Effects of guar gum content on structure and properties of multi-crosslinked polyurethane composite films," Composites Part B: Engineering, vol. 44, no. 1, pp. 76-83, 2013.

[4] C. O. Akintayo, E. T. Akintayo, T. Ziegler, and B. M. Babalola, "Newly developed epoxy-polyol and epoxy-polyurethane from renewable resources," British Journal of Applied Science \& Technology, vol. 3, no. 4, pp. 984-993, 2013.

[5] K. Yusoh, J. Jin, and M. Song, "Subsurface mechanical properties of polyurethane/organoclay nanocomposite thin films studied by nanoindentation," Progress in Organic Coatings, vol. 67 , no. 2, pp. 220-224, 2010.

[6] C. P. Krushna and P. L. Nayak, "Synthesis and characterization of polyurethane nanocomposite from castor oil-hexamethylene diisocyanate (HMDI)," Advances in Applied Science Research, vol. 5, pp. 3045-3052, 2012.

[7] D. Bhuva Bharat, Studies on some polyurethanes and fiber reinforced composites [Ph.D. thesis], Saurashtra University, 2010.

[8] X. Kong, G. Liu, and J. M. Curtis, "Novel polyurethane produced from canola oil based poly(ether ester) polyols: synthesis, characterization and properties," European Polymer Journal, vol. 48, no. 12, pp. 2097-2106, 2012.

[9] G. Lligadas, J. C. Ronda, M. Galià, and V. Cádiz, "Oleic and undecylenic acids as renewable feedstocks in the synthesis of polyols and polyurethanes," Polymers, vol. 2, no. 4, pp. 440-453, 2010.

[10] P. Cinelli, I. Anguillesi, and A. Lazzeri, "Green synthesis of flexible polyurethane foams from liquefied lignin," European Polymer Journal, vol. 49, no. 6, pp. 1174-1184, 2013.

[11] H. Fan, A. Tekeei, G. J. Suppes, and F. H. Hsieh, "Properties of biobased rigid polyurethane foams reinforced with fillers: microspheres and nanoclay," International Journal of Polymer Science, vol. 2012, Article ID 474803, 8 pages, 2012.

[12] M. Spontón, N. Casis, P. Mazo et al., "Biodegradation study by Pseudomonas sp. of flexible polyurethane foams derived from castor oil," International Biodeterioration and Biodegradation, vol. 85, pp. 85-94, 2013.

[13] C.-W. Chang and K.-T. Lu, "Natural castor oil based 2-package waterborne polyurethane wood coatings," Progress in Organic Coatings, vol. 75, no. 4, pp. 435-443, 2012.
[14] J. Xu, Z. Liu, S. Z. Erhan, and C. J. Carriere, "A potential biodegradable rubber-viscoelastic properties of a soybean oilbased composite," Journal of the American Oil Chemists' Society, vol. 79, no. 6, pp. 593-596, 2002.

[15] S. D. Baruah, "Biodegradable polymer: the promises and the problems," Science and Culture, vol. 77, pp. 11-12, 2011.

[16] C. T. Yuan, Polyurethane foams from novel soy-based polyols, The Faculty of the Graduate School at the University of Missouri, 2008.

[17] H. Yeganeh and P. Hojati-Talemi, "Preparation and properties of novel biodegradable polyurethane networks based on castor oil and poly(ethylene glycol)," Polymer Degradation and Stability, vol. 92, no. 3, pp. 480-489, 2007.

[18] M. A. Corcuera, L. Rueda, B. Fernandez D’Arlas et al., "Microstructure and properties of polyurethanes derived from castor oil," Polymer Degradation and Stability, vol. 95, no. 11, pp. 21752184,2010

[19] E. A. Ismail, A. M. Motawie, and E. M. Sadek, "Synthesis and characterization of polyurethane coatings based on soybean oilpolyester polyols," Egyptian Journal of Petroleum, vol. 20, no. 2, pp. 1-8, 2011.

[20] L.-T. Yang, C.-S. Zhao, C.-L. Dai, L.-Y. Fu, and S.-Q. Lin, "Thermal and mechanical properties of polyurethane rigid foam based on epoxidized soybean oil," Journal of Polymers and the Environment, vol. 20, no. 1, pp. 230-236, 2012.

[21] H. Bakhshi, H. Yeganeh, S. Mehdipour-Ataei, M. A. Shokrgozar, A. Yari, and S. N. Saeedi-Eslami, "Synthesis and characterization of antibacterial polyurethane coatings from quaternary ammonium salts functionalized soybean oil based polyols," Materials Science and Engineering C, vol. 33, no. 1, pp. 153-164, 2013.

[22] B. Das, U. Konwar, M. Mandal, and N. Karak, "Sunflower oil based biodegradable hyperbranched polyurethane as a thin film material," Industrial Crops and Products, vol. 44, pp. 396-404, 2013.

[23] P. Rojek and A. Prociak, "Effect of different rapeseed-oilbased polyols on mechanical properties of flexible polyurethane foams," Journal of Applied Polymer Science, vol. 125, no. 4, pp. 2936-2945, 2012.

[24] A. Fridrihsone, U. Stirna, B. Lazdiņa, M. Misane, and D. Vilsone, "Characterization of polyurethane networks structure and properties based on rapeseed oil derived polyol," European Polymer Journal, vol. 49, no. 6, pp. 1204-1214, 2013.

[25] A. S. More, T. Lebarbé, L. Maisonneuve, B. Gadenne, C. Alfos, and H. Cramail, "Novel fatty acid based di-isocyanates towards the synthesis of thermoplastic polyurethanes," European Polymer Journal, vol. 49, no. 4, pp. 823-833, 2013.

[26] M. Carme Coll Ferrer, D. Babb, and A. J. Ryan, "Characterisation of polyurethane networks based on vegetable derived polyol," Polymer, vol. 49, no. 15, pp. 3279-3287, 2008.

[27] S. R. Ivan, D. B. Zoran, and H. Berta, "Thermal stability of polyurethane materials based on castor oil as polyol component," Journal of Thermal Analysis and Calorimetry, vol. 111, no. 2, pp. 1083-1091, 2013.

[28] D. J. dos Santos, L. B. Tavares, and G. F. Batalha, "Mechanical and physical properties investigation of polyurethane material obtained from renewable natural source," Journal of Achievements in Materials and Manufacturing Engineering, vol. 54, pp. 211-217, 2012.

[29] M. Endo, M. S. Strano, and P. M. Ajayan, "Potential applications of carbon nanotubes," in Carbon Nanotubes, vol. 111 of Topics in Applied Physics, pp. 13-62, 2008. 
[30] R. V. Kurahatti, A. O. Surendranathan, S. A. Kori, N. Singh, A. V. R. Kumar, and S. Srivastava, "Defence applications of polymer nanocomposites," Defence Science Journal, vol. 60, no. 5, pp. 551563,2010

[31] Y.-L. Luo, B.-X. Wang, and F. Xu, "Effect of soft-hard segment structure on vapor responsiveness of polyurethane conducting composite thin films loaded with multi-walled carbon nanotubes," Sensors and Actuators B: Chemical, vol. 156, no. 1, pp. 12-22, 2011.

[32] S. K. Yadav and J. W. Cho, "Functionalized graphene nanoplatelets for enhanced mechanical and thermal properties of polyurethane nanocomposites," Applied Surface Science, vol. 266, pp. 360-367, 2013.

[33] T. M. Madkour, F. M. Hagag, W. Mamdouh, and R. A. Azzam, "Molecular-level modeling and experimental investigation into the high performance nature and low hysteresis of thermoplastic polyurethane/multi-walled carbon nanotube nanocomposites," Polymer, vol. 53, no. 25, pp. 5788-5797, 2012.

[34] S. Yun, H. Im, and J. Kim, "The effect of different hard segments in polyurethane on the electrical conductivity of polyurethane grafted multi-walled carbon nanotube/polyurethane nanocomposites," Synthetic Metals, vol. 161, no. 13-14, pp. 1361-1367, 2011.

[35] R. Manivannan, A. Daniel, I. Srikanth et al., "Thermal stability of zirconia-coated multiwalled carbon nanotubes," Defence Science Journal, vol. 60, no. 3, pp. 337-342, 2010.

[36] R. N. Jana, H. J. Yoo, and J. W. Cho, "Synthesis and properties of shape memory polyurethane nanocomposites reinforced with poly( $\varepsilon$-caprolactone)-grafted carbon nanotubes," Fibers and Polymers, vol. 9, no. 3, pp. 247-254, 2008.

[37] R. Yudianti, H. Onggo, Y. Saito, T. Iwata, and J.-I. Azuma, "Analysis of functional group sited on multi-wall carbon Nanotube surface," Open Materials Science Journal, vol. 5, pp. 242-247, 2011.

[38] S. K. Yadav, S. S. Mahapatra, and J. W. Cho, "Synthesis of mechanically robust antimicrobial nanocomposites by click coupling of hyperbranched polyurethane and carbon nanotubes," Polymer, vol. 53, no. 10, pp. 2023-2031, 2012.

[39] S. Gu, B. Yan, L. Liu, and J. Ren, "Carbon nanotube-polyurethane shape memory nanocomposites with low trigger temperature," European Polymer Journal, vol. 49, no. 12, pp. 3867-3877, 2013.

[40] N. G. Sahoo, Y. C. Jung, H. H. So, and J. W. Cho, "Synthesis of polyurethane nanocomposites of functionalized carbon nanotubes by in-situ polymerization methods," Journal of the Korean Physical Society, vol. 51, pp. 1-6, 2007.

[41] X. Wang, Z. Du, C. Zhang, C. Li, X. Yang, and H. Li, "Multiwalled carbon nanotubes encapsulated with polyurethane and its nanocomposites," Journal of Polymer Science A: Polymer Chemistry, vol. 46, no. 14, pp. 4857-4865, 2008.

[42] K. B. Aruna and K. T. Deba, "Preparation, characterization and properties of acid functionalized multi-walled carbon nanotube reinforced thermoplastic polyurethane nanocomposites," Materials Science and Engineering B: Solid-State Materials for Advanced Technology, vol. 176, no. 18, pp. 1435-1447, 2011.

[43] H.-C. Kuan, C.-C. M. Ma, W.-P. Chang, S.-M. Yuen, H.-H. $\mathrm{Wu}$, and T.-M. Lee, "Synthesis, thermal, mechanical and rheological properties of multiwall carbon nanotube/waterborne polyurethane nanocomposite," Composites Science and Technology, vol. 65, no. 11-12, pp. 1703-1710, 2005.

[44] O. Zhou, R. M. Fleming, D. W. Murphy et al., "Defects in carbon nanostructures," Science, vol. 263, no. 5154, pp. 1744-1747, 1994.
[45] P.-C. Ma, N. A. Siddiqui, G. Marom, and J.-K. Kim, "Dispersion and functionalization of carbon nanotubes for polymer-based nanocomposites: a review," Composites A: Applied Science and Manufacturing, vol. 41, no. 10, pp. 1345-1367, 2010.

[46] L. Nayak, M. Rahaman, D. Khastgir, and T. K. Chaki, “Thermal and electrical properties of carbon nanotubes based polysulfone nanocomposites," Polymer Bulletin, vol. 67, no. 6, pp. 1029-1044, 2011.

[47] H. J. Yoo, Y. C. Jung, N. G. Sahoo, and J. W. Cho, "Polyurethanecarbon nanotube nanocomposites prepared by in-situ polymerization with electroactive shape memory," Journal of Macromolecular Science B: Physics, vol. 45, no. 4, pp. 441-451, 2006.

[48] K. Wongtimnoi, B. Guiffard, A. Bogner-van de Moortèle, L. Seveyrat, and J.-Y. Cavaillé, "Electrostrictive thermoplastic polyurethane-based nanocomposites filled with carboxylfunctionalized multi-walled carbon nanotubes (MWCNT$\mathrm{COOH})$ : properties and improvement of electromechanical activity," Composites Science and Technology, vol. 85, pp. 23-28, 2013.

[49] S. Guo, C. Zhang, W. Wang et al., "Preparation and characterization of polyurethane/multiwalled carbon nanotube composites," Polymers and Polymer Composites, vol. 16, no. 8, pp. 471478, 2008.

[50] N. G. Sahoo, Y. C. Jung, H. H. So, and J. W. Cho, "Synthesis of polyurethane nanocomposites of functionalized carbon nanotubes by in-situ polymerization methods," Journal of the Korean Physical Society, vol. 51, no. 1, 2007.

[51] P. K. Maji, N. K. Das, and A. K. Bhowmick, "Preparation and properties of polyurethane nanocomposites of novel architecture as advanced barrier materials," Polymer, vol. 51, no. 5, pp. 1100-1110, 2010.

[52] M. Joshi, K. Banerjee, R. Prasanth, and V. Thakare, "Polymer/clay nanocomposite based coatings for enhanced gas barrier property," Indian Journal of Fibre and Textile Research, vol. 31, no. 1, pp. 202-214, 2006.

[53] S. Brunauer, P. H. Emmett, and E. Teller, "Adsorption of gases in multimolecular layers," Journal of the American Chemical Society, vol. 60, no. 2, pp. 309-319, 1938. 

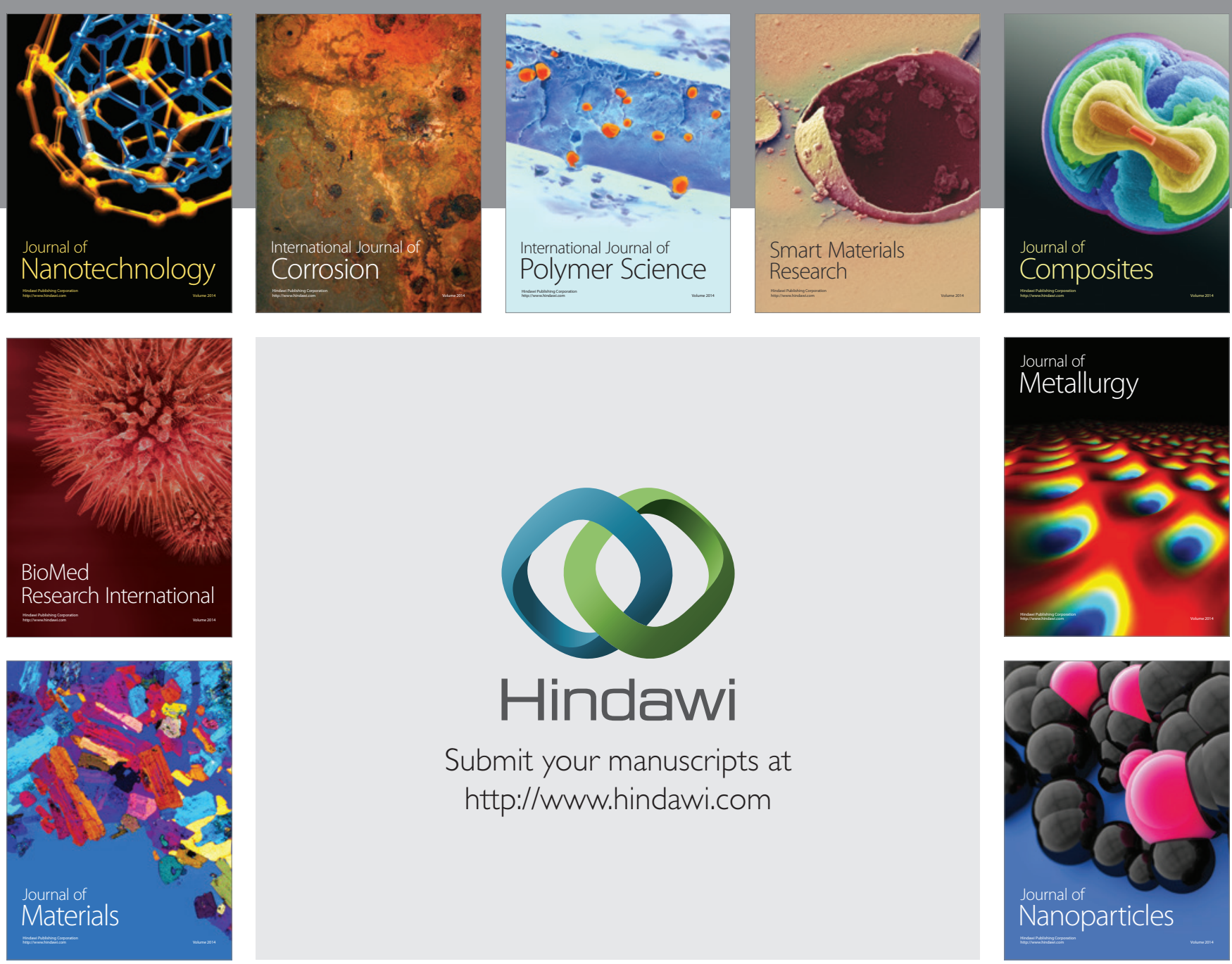

Submit your manuscripts at http://www.hindawi.com
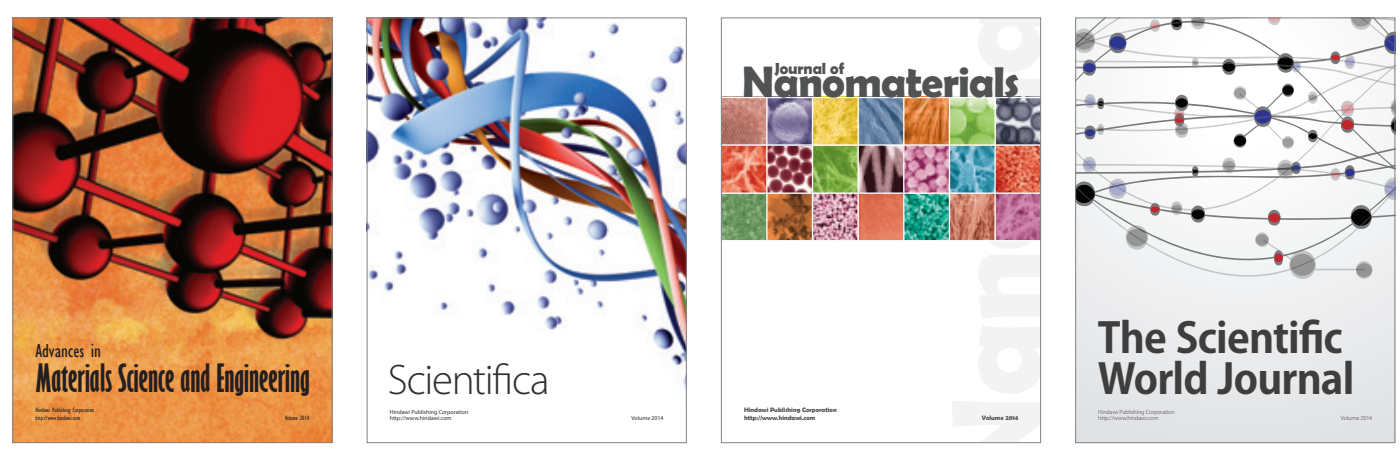

\section{The Scientific World Journal}
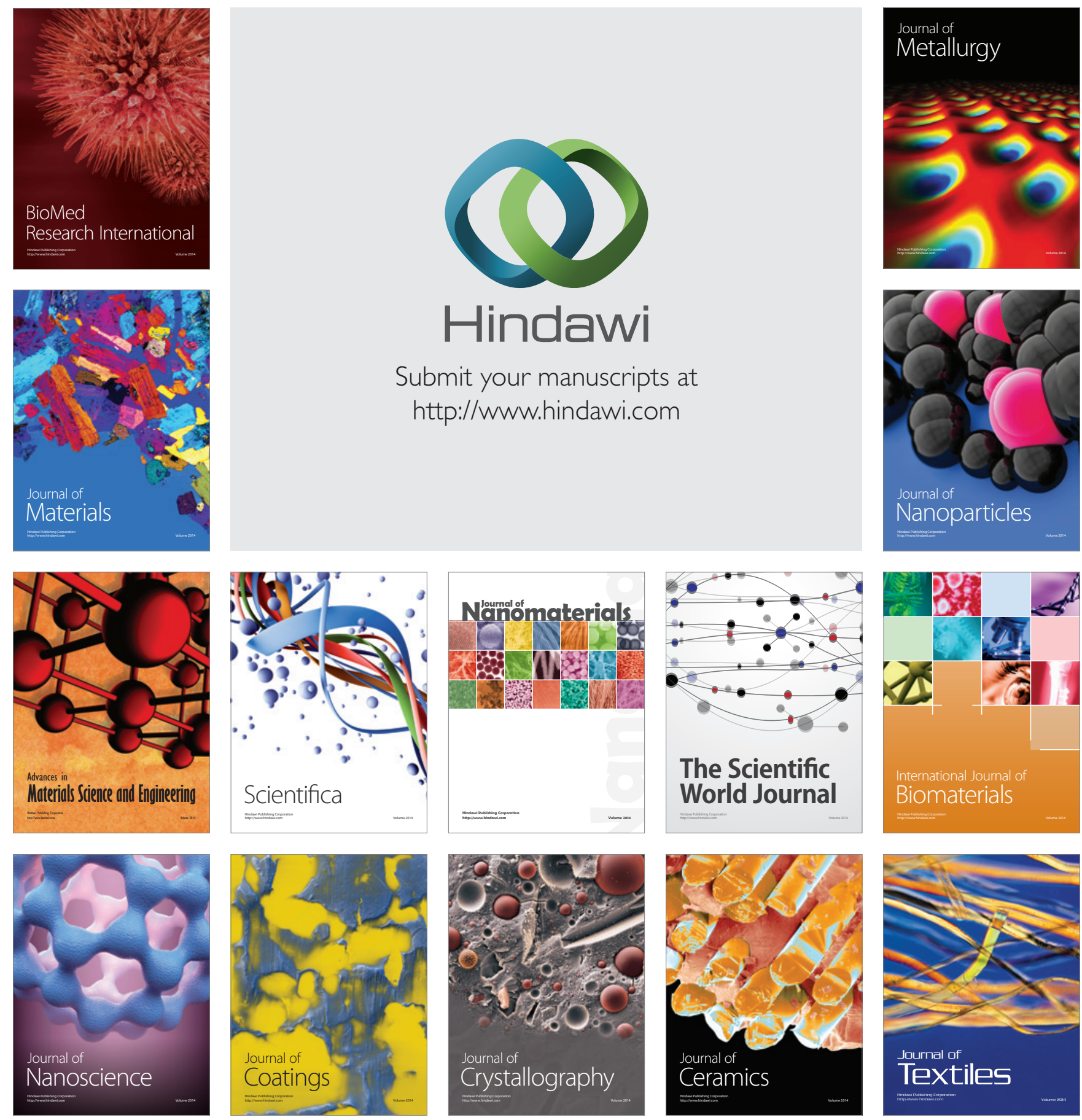